\title{
Testing our tests: Do clinical studies of diagnostic performance truly inform patient management?
}

\author{
Prem Soman, MD, PhD, FASNC, ${ }^{a}$ and Matthew E. Harinstein, MD, FASNC ${ }^{a}$ \\ a Division of Cardiology and The Heart and Vascular Institute, University of Pittsburgh Medical \\ Center, Pittsburgh, PA
}

Received Jan 17, 2018; accepted Jan 18, 2018

doi: $10.1007 / \mathrm{s} 12350-018-1210-2$

\section{See related article, pp. 1269-1279}

In this issue of the Journal, we are presented with a report by Barone-Rochette et al., of the diagnostic accuracy of a novel approach to myocardial perfusion imaging for stable coronary artery disease (CAD). ${ }^{1}$ The protocol utilized was dual isotope, stress thallium-201/ rest Tc-99m. The dose was $2 \mathrm{mCi}$ of Tl-201, and around $8 \mathrm{mCi}$ of Tc-99m sestamibi for patients $<80 \mathrm{Kg}$, and weight-based for heavier patients, resulting in an average total body effective dose of $12 \mathrm{mSv}$. Strengths of the study include the use of FFR, and not just visually interpreted coronary angiography as the comparator standard, and the fact that all patients were referred for coronary angiography. While the latter fact avoids the verification bias resulting from selective angiography in patients with abnormal scans, it introduces a bias of its own by selecting only high-risk patients as subjects for the study. This selection bias was reflected in the results, with reported patient-level sensitivity, specificity, and diagnostic accuracy of $92.8 \%, 69.2 \%$, and $81.4 \%$, respectively.

The protocol is novel in that it used the strengths of solid-state (Cadmium Zinc Telluride-crystal) technology to enable low-dose imaging with $\mathrm{Tl}-201$, a tracer that has a higher extraction fraction than Tc-99m sestamibi or Tc-99m tetrofosmin, and thus might be expected to improve the sensitivity of detecting mild disease and

Reprint requests: Prem Soman, MD, PhD, FASNC Division of Cardiology and The Heart and Vascular Institute, University of Pittsburgh Medical Center, A-429 Scaife Hall, 200 Lothrop Street, Pittsburgh, PA 15213; premsoman@usa.net

J Nucl Cardiol 2019;26:1284-5.

$1071-3581 / \$ 34.00$

Copyright (c) 2018 American Society of Nuclear Cardiology. identify more ischemia. While this logic is based on sound physiological concept, ${ }^{2}$ the study did not actually demonstrate that this is true by comparing this protocol to a single-isotope, Tc-99m-based one. Thus, it remains mostly a feasibility study of a dual isotope protocol using low-dose thallium-201. In this respect, it is not exclusive, since such an approach has been proven feasible before. ${ }^{3}$ In fact, like many similar studies of diagnostic performance, the manuscript leaves the reader with many unanswered questions:

1. Can the results of this study, generated in a small patient population $(n=54)$ with a high suspicion of CAD (already referred for coronary angiography) be extrapolated to a broad population of stable patients with known or suspected CAD?

2. Is the protocol effective in real-life situations? While comparative studies generally assess diagnostic accuracy parameters like sensitivity and specificity (efficacy) under controlled circumstances, how a test performs in real life (effectiveness) is important from a pragmatic standpoint. For example, studies show that CT coronary angiography (CTCA) has a higher negative predictive value than other imaging tests for CAD. ${ }^{4}$ However, CTCA may not be the optimal test in patients with contrast allergy, renal dysfunction, atrial fibrillation, and tachycardia, and furthermore, may not be available to physicians in a particular setting. Similar considerations apply to stress cardiac magnetic resonance (CMR). How effective a test is in a particular population, i.e., how many patients referred to a test actually get it, is rarely addressed in a clinical study. Effectiveness is an underappreciated attribute of stress SPECT, a test that is widely available and can be applied to broad populations of patients irrespective of body habitus, metallic devices, heart rate, atrial fibrillation, and renal 
failure. Thus, most patients referred to stress SPECT imaging actually get it.

3. Is the higher radiation dose compared to a low-dose, single-isotope Tc-99m protocol justified by a higher diagnostic accuracy and more accurate quantification of myocardial ischemia?

4. Most importantly, does the choice of this protocol in preference to others have an impact on relevant outcome measures? In the current environment of value-based care, this is an important consideration. A case in point is the study by Siebelink and colleagues, comparing SPECT (stress/ rest Tc-99m sestamibi) and PET (N-13 NH3 and F-18 FDG) for viability assessment in 103 patients with moderate LV systolic dysfunction. While it is generally accepted that PET detects viability more efficiently than SPECT, this study showed that treatment allocation (revascularization vs. medical therapy alone) and patient outcome were not impacted by the choice of modality (SPECT or PET-based information provided to blinded treating physicians in random order). ${ }^{5}$ Comparative studies of diagnostic tests should aim to demonstrate whether improvements in diagnostic performance translate into meaningful clinical advantage.

The last decade saw a profusion of comparative diagnostic imaging studies in stable CAD populations, resulting from burgeoning new diagnostic modalities and expanding applications of existing ones. Studies such as the current one by Barone-Rochette and colleagues provide important insights into the performance characteristics of diagnostic tests for stable CAD. However, to truly inform our management of patients in an additive manner, studies should be designed to provide information beyond just diagnostic accuracy.

\section{Disclosure}

Dr. Soman is the PI of an investigator-initiate research grant from Astellas to the University of Pittsburgh. Dr. Harinstein has no disclosure relevant to this publication.

\section{References}

1. Barone -Rochette G, Zoreka F, Djaileb L, Pileiro N, Calizzano A, Quesada JL, Broisat A, Rious L, Machecout J, Fagret D, Vanzetto G, Gezzi C. Diagnostic value of stress Thallium-201/Rest Technetium-99m sestamibi sequential dual isotope high-speed myocardial perfusion imaging for the detection of hemodynamically significant coronary artery stenosis. J Nucl Cardiol 2018.

2. Soman P, Taillefer R, DePuey EG, Udelson JE, Lahiri A. Enhanced detection of reversible perfusion defects by Tc-99m sestamibi compared to Tc-99m tetrofosmin during vasodilator stress SPECT imaging in mild-to-moderate coronary artery disease. J Am Coll Cardiol 2001;37:458-62.

3. Berman DS, Kang X, Tamarappoo B, et al Stress thallium-201/rest technetium-99m sequential dual isotope high-speed myocardial perfusion imaging. JACC Cardiovasc Imaging 2009;2:273-82.

4. Chow BJ, Abraham A, Wells GA, et al Diagnostic accuracy and impact of computed tomographic coronary angiography on utilization of invasive coronary angiography. Circ Cardiovasc Imaging 2009;2:16-23.

5. Siebelink HM, Blanksma PK, Crijns HJ, et al No difference in cardiac event-free survival between positron emission tomographyguided and single-photon emission computed tomography-guided patient management: a prospective, randomized comparison of patients with suspicion of jeopardized myocardium. J Am Coll Cardiol 2001;37:81-8. 